\title{
RESEARCH AND APPLICATION OF LiDAR TECHNOLOGY IN CADASTRAL SURVEYING AND MAPPING
}

\author{
G. B. He, L. L. Li \\ Aerial Photogrammetry and Remote Sensing Group Co. LTD Of China National Administration of Coal Geology (ARSC), Xi'an, \\ China, 1llcug@163.com
}

KEY WORDS: LiDAR Scanning Technology, UAV, Point Cloud Data, Cadastral Surveying and Mapping

\begin{abstract}
:
The state attaches great importance to the issue of the right to the use of rural land. At present, China is carrying out a large-scale mapping and confirmation of rural house sites and rural houses. The traditional measurement method mainly adopts total station or RTK manual measurement method, which is characterized by high work intensity, complex operation procedure and laborconsuming. There are hundreds of thousands of villages in China. With the development of science and technology, it is an inevitable trend to use new technologies in rural cadastral surveys to improve their efficiency and quality. LiDAR scanning technology has the advantages of high efficiency, flexibility, reliability, and high accuracy, which meet the requirements of tasks and schedules that are difficult to complete with traditional measurement methods. This paper is based on the AS-300H multi-platform LiDAR system of Huace Navigation, which integrates UAV and vehicle LiDAR for cadastral surveying and mapping. It proves that LiDAR using in rural cadastral mapping has low labor intensity, high efficiency and accuracy. At the same time, the advantages and disadvantages of LiDAR technology for cadastral mapping are also summarized and elaborated, which has a good reference and significance for those who are currently conducting rural housing and cadastral mapping work.
\end{abstract}

\section{INTRODUCTION}

At present, in order to promote the reform of the rural property rights system, China is carrying out large-scale investigations, mapping, and ownership confirmation and registration of rural homesteads and houses (Independent Multidisciplinary Science Team, 2012). In this work, the most basic and important is the rural cadastral and real estate mapping work. Because this work involves the immediate rights and interests of farmers, the technical requirements for rural cadastral mapping are very high. According to the current cadastral surveying and mapping specifications in China, the mean square error of the plane position measurement of the boundary point is less than $5 \mathrm{~cm}$ (Zhang J, et al., 2017). The traditional surveying methods mainly use total stations or GNSS equipment to collect and measure the coordinates of cadastral or house. However, the efficiency of this method is very low, and the mapping quality is difficult to control (Cina A, et al., 2016) and (Abidin H Z, et al., 2015). There are hundreds of thousands of villages in China. If the traditional method is used for mapping, the time and cost required will be unacceptable. Therefore, there is an urgent need to find a more efficient rural cadastral mapping method to improve work efficiency.

In recent years, with the popularity of unmanned airborne vehicle (UAV) and the maturity of micro-airborne Light Detection and Ranging (LiDAR) surveying and mapping equipment and data processing technology, many surveying and mapping workers have begun to use the UAV LiDAR technology to carry out rural cadastral surveying and mapping (Bao J, 2019) and (In et al., 2011). In the traditional concept, the accuracy of aerial survey mapping cannot meet the accuracy requirements of cadastral mapping. In this paper, the analysis and research of pilot projects for rural cadastral surveying and mapping using integrated UAV and vehicle LiDAR methods have fully proved the feasibility of LiDAR technology for rural cadastral surveying and mapping in the northern plains of China. Due to the use of high-precision POS system and very low flight altitude, and the integration of UAV and vehicle LiDAR methods to carry out rural cadastral mapping, the accuracy of the results can fully meet the accuracy requirements of 1:500 rural cadastral mapping. Moreover, the method has many advantages, such as low labor intensity, high efficiency, high measurement accuracy, rich data results and so on (Ussyshkin $\mathrm{R}$ V, et al., 2011.) and (Thiel $C$ et al., 2017), which is a very worthy method of rural cadastral mapping.

At present, cadastral surveying and mapping in China is based on ownership surveys, with the purpose of obtaining and expressing cadastral information, involving survey control cadastral, survey cadastral elements, survey cadastral elements and so on. In order to ensure the efficiency of Surveying and mapping, we should pay attention to the principles from high level to low level, from whole to part and from control to detail (Gao Wenxiu, 1999.). At the same time, China has a vast territory and a large amount of cadastral surveying work. Therefore, the application of professional technology to continuously improve the quality and efficiency of cadastral surveying and mapping is the focus of national functional departments. 3D scanning technology can achieve a large number of acquisition of $3 \mathrm{D}$ points, and scan a variety of complex environments, collect irregular, high complexity, large-scale real-world 3D data, and use the computer and related software to achieve the construction of the target 3D point cloud model. The application to the foundation surveying and mapping work helps to better grasp the geographic element information of the target to be measured, as well as the coordinates of the boundary point of the land ownership boundary, and provides an important basis for formulating related strategies.

\section{ADVANTAGES AND APPLICATION PROCESS OF LIDAR SURVEY TECHNOLOGY IN RURAL CADASTRAL MAPPING}

\subsection{Advantages of LiDAR Surveying Technology in Rural Cadastral Surveying and Mapping}

LiDAR is an advanced detection method combining laser technology with modern photoelectLiDAR is an advanced detection method combining laser technology with modern 
photoelectric detection technology, which can obtain 3D point clouds of ground objects. LiDAR can carry out multi platform carrying, including manual carrying, airborne, on-board and onboard (Zhou Z W, 2011). LiDAR survey technology has the following advantages in the application of rural cadastral mapping:

(1) LiDAR technology has low requirements for light, and can collect data during the day and night. It can make full use of the evening work with better weather and improve the efficiency of data acquisition;

(2) There is no need to arrange image control points,greatly reducing the field work period;

(3) In the case of less severe occlusion, due to the multi-echo characteristics of laser scanning, the wall surface of the house is intact, and there will be no deformation in the periphery, which can improve the accuracy of the data.

(4) The boundary of the house is presented in the form of a vector point cloud, which can more intuitively determine the position of the wall and reduce the judgment process.

\subsection{Application Process of LiDAR Survey Technology in Rural Cadastral Mapping}

In order to verify the effect of LiDAR measurement technology in cadastral surveying and mapping, based on the LiDAR system of Huace AS-300H multi platform, this paper mainly obtains the LiDAR point cloud of UAV, and uses the vehicle LiDAR method to supplement and acquire the point cloud data in the area where there is occlusion and cannot be obtained. The rural cadastral mapping is carried out by combining the data of LiDAR point cloud acquired by UAV and vehicle. In field work, the accuracy of LiDAR survey technology used in rural cadastral mapping is analyzed by comparing LiDAR point cloud data with total station data. The specific operation process is shown in the figure below:

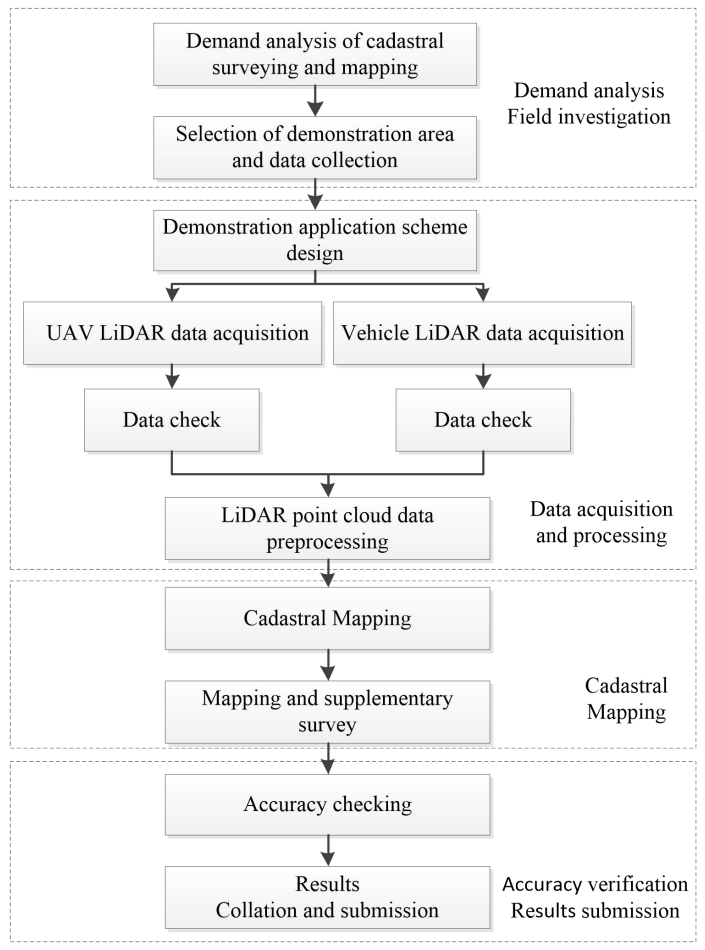

Figure 1 The workflow of this paper.

\section{THE SPECIFIC APPLICATION AND ANALYSIS OF LIDAR MEASUREMENT TECHNOLOGY IN CADASTRAL SURVEYING AND MAPPING}

\subsection{Survey Area}

An area of about $0.5 \mathrm{~km}^{2}$ around the health center of Wujiazhai village, Wuyi District, Xi'an City, China was selected as the experimental area for rural cadastral surveys with integrated UAV airborne and vehicle-mounted laser measurement systems. Wujiazhai Village is located in the west of the southern district of Xi'an, $34^{\circ} 06^{\prime} 59^{\prime \prime} \sim 34^{\circ} 07^{\prime} 11^{\prime \prime} \mathrm{N}, 108^{\circ} 39^{\prime} 04^{\prime \prime} \sim 108^{\circ}$ $39^{\prime} 35^{\prime \prime} \mathrm{E}$. The villages in this area have flat terrain and dense houses. Road regulation is very representative in the plain area of northern China. The village houses are densely built, and the phenomenon of rowing and private sunshades is more serious. It is quite common for residents to plant walnut and other fruit trees at the door. The specific distribution is shown in Figure 2. The specific environment around the house is shown in Figure 3.

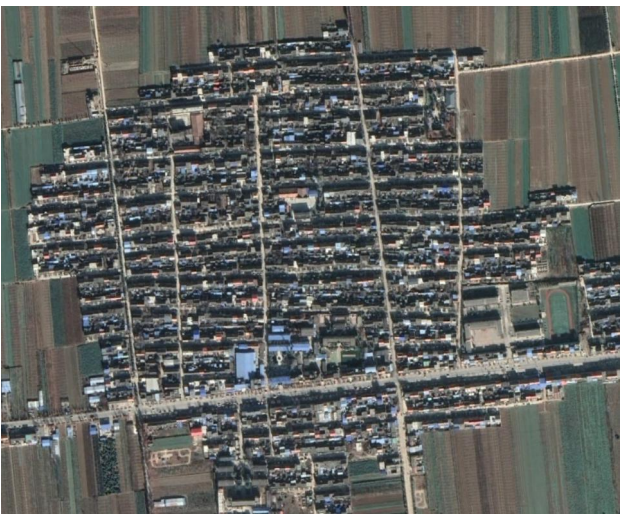

Figure 2 Location of test area.

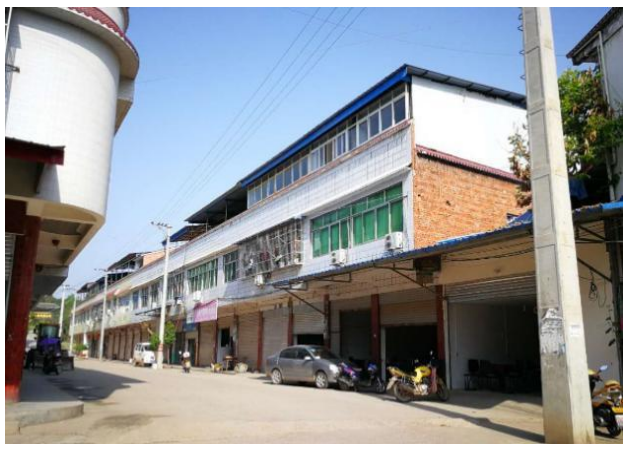

Figure 3 Housing environment.

\subsection{Lidar Data Acquisition}

3.2.1 Route Planning: Before the UAV carries out flight data collection, it should know the details of the survey area in advance, including the height of surrounding floors, the distribution and height of high-voltage power lines, the height and location of large communication base stations and other information. On the premise of ensuring the safety of airspace, set the flight route of UAV on site. According to the collection requirements of point cloud density not less than $100 \mathrm{pts} / \mathrm{m}^{2}$, the flight height of the planned route is set as $50 \mathrm{~m}$, the overlap between routes is $30 \%$, the route length is about $3300 \mathrm{~m}$, and the flight speed is $5.5 \mathrm{~m} / \mathrm{s}$.

Vehicle LiDAR is mainly used to collect the object data of the street and its two sides, which is the supplement and check of the data collected by UAV. According to the actual distribution 
and occlusion of the buildings in the test area, as well as the distribution of the road, the route to obtain the LiDAR point cloud is designed.

3.2.2 Layout of Ground Reference Base Station: In this experiment, IMU / GNSS was used to assist in obtaining LiDAR point cloud data. According to experience, each GNSS reference base station in plain area can cover an area with a radius of no less than $5 \mathrm{~km}$. The ground reference base station of the test area is set up at a known measuring point about $2.6 \mathrm{~km}$ away from the center point of the measuring area. The area of the test area is not large (about $0.5 \mathrm{~km}^{2}$ ), so it can meet the requirement of 1:500 cadastral survey accuracy to set up a ground base station. The reference base station adopts the static continuous observation mode, which requires the data sampling frequency of $1 \mathrm{~Hz}$ and the data storage format of RINEX 3.02 or $\mathrm{HCN}$. During the data collection of reference base station, the whole process is guarded by operators to prevent any accidents, so as to ensure the continuity and accuracy of base station data.

3.2.3 Field Data Collection: According to the designed technical plan, the ground and house data collection in the test area will be divided into two parts. First, UAV LiDAR data collection is the main method of data collection, which can obtain the location information of most houses and features; The other is vehicle LiDAR data acquisition, which is an auxiliary acquisition mode, mainly used to supplement part of the data that the UAV cannot acquire due to the shelter of eaves, trees, etc.

As the multi platform LiDAR measurement system AS-300H uses GNSS positioning, whether it is the UAV LiDAR data acquisition or the vehicle LiDAR data acquisition, before data acquisition, it is necessary to find an open area far away from buildings and dense vegetation outside the measurement area to conduct GNSS star searching positioning and complete equipment initialization. Under normal circumstances, in order to ensure high-precision data acquisition requirements, the number of satellites shall be at least 14 .

In this experiment, DJI M-600 Pro six-rotor UAV is equipped with AS-300H multi-platform LiDAR system to obtain point cloud data. After the UAV initialization, start the UAV. The UAV will take off vertically and enter the route autonomously, and fly according to the preset flight route. The UAV will automatically start LiDAR data collection after entering the route. During the flight, the operator should always pay attention to the flight attitude and altitude of the UAV on the flight console to ensure the flight safety of the UAV. After the acquisition flight, first check whether the flight route covers the whole survey area, and if there is a loophole, make up the flight in time to ensure the integrity of the collected data.

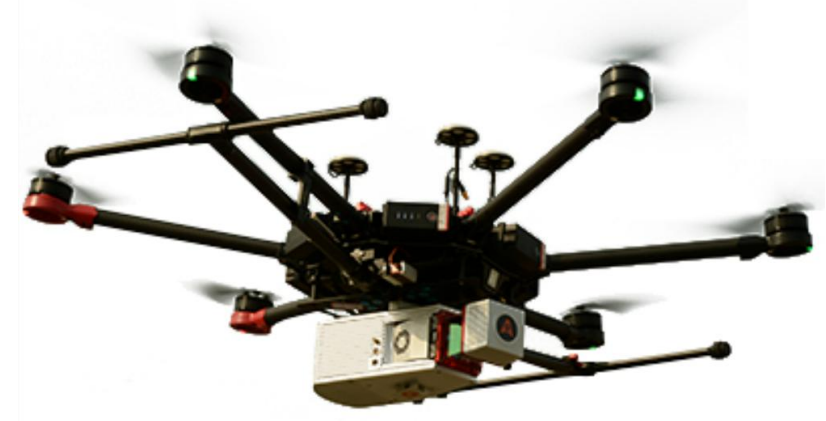

Figure 4 Dajiang M600 Pro UAV with LiDAR AS-300H.
After the data acquisition of the UAV LiDAR is completed, we will remove the LiDAR equipment from the UAV and install it on the trunk of the car to implement the vehicle LiDAR data acquisition. Vehicle LiDAR is mainly used to collect the object data of the street and its two sides, which is the supplement and check of the data collected by UAV.

After initialization of vehicle LiDAR, LiDAR equipment can be started and vehicle data acquisition can be started. The on-board LiDAR system slowly drives into the survey area from the initialization point outside the survey area, and collects data according to the on-board collection route designed in the early stage of the project. It is required that the vehicle drives stably, the driving speed is not more than $30 \mathrm{~km} / \mathrm{h}$, and it is generally not allowed to reverse. After the completion of the collection, first check whether the collection route and collection area are complete. If there are any loopholes, make up the test in time to ensure the integrity of the collected data.

After the LiDAR data collection is completed, the collected original data must be exported to the computer and backed up in time to prevent data destruction or loss.

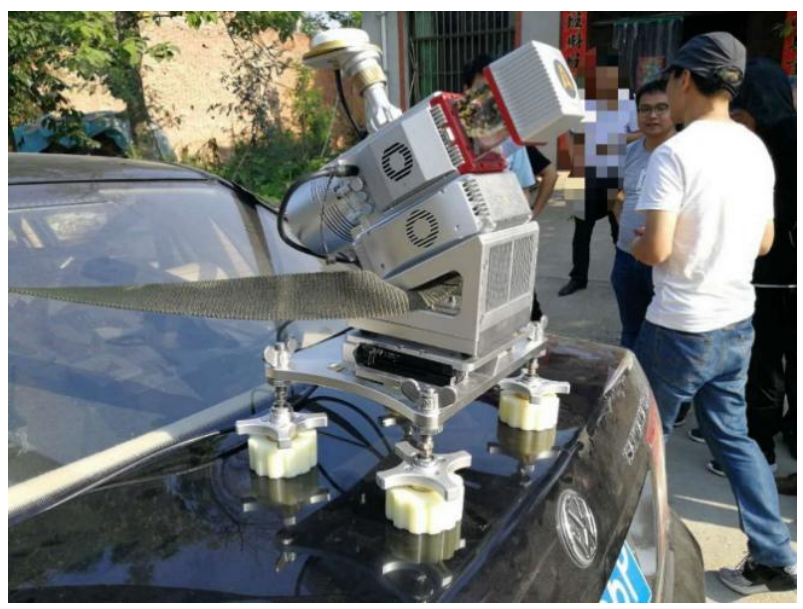

Figure 5 Vehicle point cloud data acquisition system.

\subsection{Data Processing}

3.3.1 Point Cloud Data Processing: Because the same LiDAR equipment is used on the UAV and the vehicle, the processing methods and processes of the LiDAR data collected by the UAV flight and the LiDAR data collected by the vehicle are completely the same. It mainly includes GNSS reference base station data conversion, POS solution, point cloud data preprocessing and other steps.

Since the GNSS reference base station observation uses the Huace i80 dual-frequency multi-channel GNSS receiver, the Huace Navigation CHCData software is used to output the reference base station observation data and format conversion. The process mainly includes the steps of GNSS receiver antenna height correction, RINEX3.02 format setting and file format conversion.

The trajectory and attitude (POS) of LiDAR acquisition equipment play a decisive role in the accurate calculation of measurement data. The POS solution software used in this test is Novatel Inertial Explorer 8.7.

Point cloud data preprocessing is to transform the original laser measurement point data collected by LiDAR equipment into laser point cloud data with three-dimensional coordinate 
information in the WGS84 coordinate system. Through the fusion of the high-precision trajectory data calculated by ie8.7 and the high-precision point and position measurement data collected by the laser scanner, the point cloud data with accurate three-dimensional coordinates is finally obtained. Point cloud data preprocessing adopts the copre point cloud data preprocessing software of Huace navigation. After preprocessing, we can use different rendering methods to browse point cloud data. The point cloud data rendered in elevation mode is shown in the following figure.

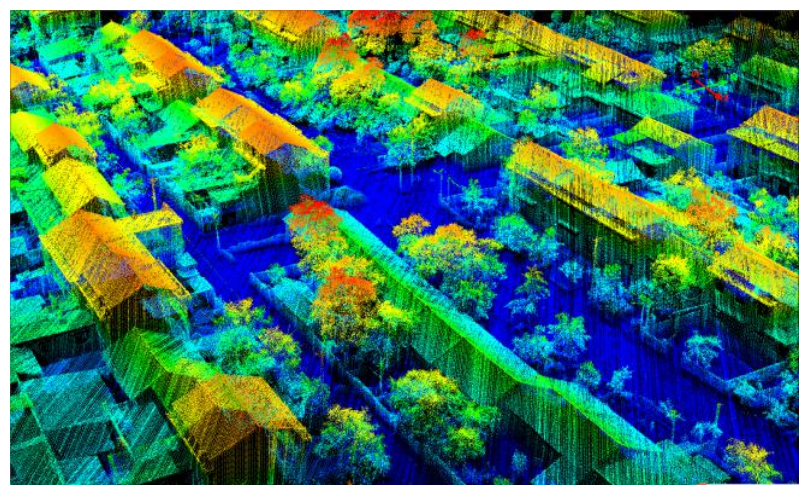

Figure 6 Point cloud data displayed with elevation.

3.3.2 Coordinate Conversion: Because the POS of LiDAR point cloud data acquisition equipment is WGS84 coordinate system, and the coordinate system required for cadastral achievement data is CGCS2000 coordinate system, it is necessary to conduct coordinate transformation for point cloud data directly obtained. The area of the test project is small. Five groups of measurement points are selected around and in the center of the test area, and WGS 84 coordinates and CGCS2000 coordinates are measured at the same time. The four parameters applicable to the test area are calculated with these 5 groups of points, and then the point cloud results are uniformly converted to CGCS2000 coordinate system.

3.3.3 Cadastral Mapping: After obtaining the threedimensional point cloud data file in Las format through preprocessing, the CoMapping cadastral mapping software of Huace Navigation Company is used to collect and draw the cadastral elements based on the intuitive point cloud model.

CoMapping software is based on true $3 \mathrm{D}$ stereo environment mapping, supporting point cloud data, orthophoto data, and is seamlessly compatible with traditional aerial survey operations. During the operation, encountering incomplete point cloud scanning on a certain wall, and locating the point cloud by point cloud slicing to make it easier to draw. Even corner points that are not easy to capture can be drawn using line intersections. The partial results as shown in Figure 7 and Figure 8.

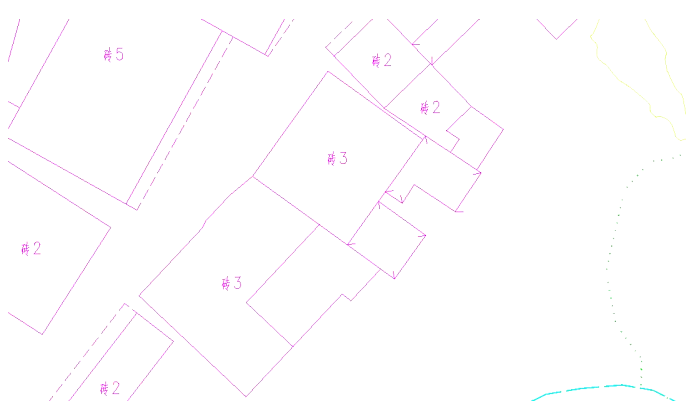

Figure 7 The partial results of cadastral map.

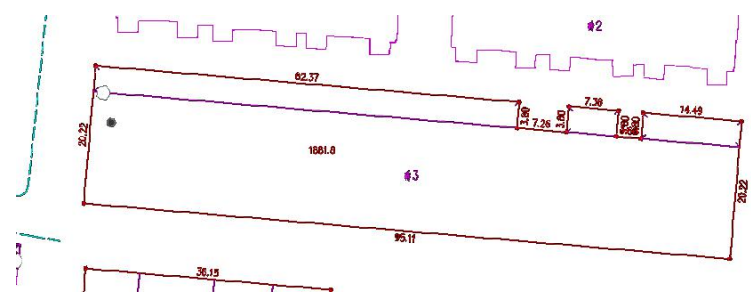

Figure 8 The partial results of cadastral map.

\subsection{Results Accuracy Verification}

The accuracy requirements of cadastral survey are relatively strict, which requires that the measurement accuracy of various house corner points as boundary sites should meet the requirement that the mean square error of point plane coordinates is $\leq 5 \mathrm{~cm}$. In this experiment, using traditional GNSS and total station measurement methods to collect some feature points and compare them with LiDAR methods to determine the accuracy of LiDAR measurements. The plane coordinates of 25 checkpoints were collected in the field in this experimental area, and they were used as the true values to test the same-named points measured by LiDAR. Based on the acquired LiDAR point cloud data, the coordinates of feature points in the cadastral point cloud data in the experimental area were collected. The coordinates of the corresponding feature points measured in the field are imported into the same system, and then the coordinates of the two sets of corresponding feature points are compared to calculate the measurement accuracy, and the application effect of LiDAR technology in cadastral survey is verified. The test results are shown in Table 1 below. Among them, the plane error of 17 points is less than $5 \mathrm{~cm}$, the plane error of 8 points is $5-10 \mathrm{~cm}$, and the plane mean square error is $\pm 0.047 \mathrm{~m}$, which fully meets the requirements of cadastral surveying and mapping specifications.

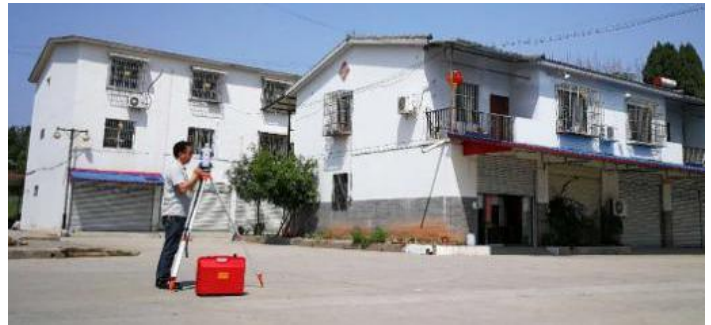

Figure 9 Measurement trajectory.

\begin{tabular}{ccc|ccc}
\hline $\begin{array}{c}\text { Serial } \\
\text { Number }\end{array}$ & $\begin{array}{c}\text { Plane } \\
\text { Error }\end{array}$ & $\begin{array}{c}\text { Error } \\
\text { Attribute }\end{array}$ & $\begin{array}{c}\text { Serial } \\
\text { Number }\end{array}$ & $\begin{array}{c}\text { Plane } \\
\text { Error }\end{array}$ & $\begin{array}{c}\text { Error } \\
\text { Attribute }\end{array}$ \\
\hline 1 & 0.045 & $<5 \mathrm{~cm}$ & 14 & 0.079 & $5-10 \mathrm{~cm}$ \\
2 & 0.033 & $<5 \mathrm{~cm}$ & 15 & 0.049 & $<5 \mathrm{~cm}$ \\
3 & 0.051 & $5-10 \mathrm{~cm}$ & 16 & 0.059 & $5-10 \mathrm{~cm}$ \\
4 & 0.042 & $<5 \mathrm{~cm}$ & 17 & 0.073 & $5-10 \mathrm{~cm}$ \\
5 & 0.051 & $5-10 \mathrm{~cm}$ & 18 & 0.013 & $<5 \mathrm{~cm}$ \\
6 & 0.057 & $5-10 \mathrm{~cm}$ & 19 & 0.074 & $5-10 \mathrm{~cm}$ \\
7 & 0.016 & $<5 \mathrm{~cm}$ & 20 & 0.024 & $<5 \mathrm{~cm}$ \\
8 & 0.039 & $<5 \mathrm{~cm}$ & 21 & 0.030 & $<5 \mathrm{~cm}$ \\
9 & 0.007 & $<5 \mathrm{~cm}$ & 22 & 0.048 & $<5 \mathrm{~cm}$ \\
10 & 0.047 & $<5 \mathrm{~cm}$ & 23 & 0.021 & $<5 \mathrm{~cm}$ \\
11 & 0.026 & $<5 \mathrm{~cm}$ & 24 & 0.019 & $<5 \mathrm{~cm}$ \\
12 & 0.078 & $5-10 \mathrm{~cm}$ & 25 & 0.034 & $<5 \mathrm{~cm}$ \\
13 & 0.049 & $<5 \mathrm{~cm}$ & & & \\
\hline \multicolumn{5}{c}{ Mean square error of plane position: } & $\pm \mathbf{0 . 0 4 7 m}$ \\
\hline
\end{tabular}

Table 1 The comparison of accuracy of point cloud coordinates and detection point coordinates. 


\subsection{Operational Efficiency Analysis}

In order to evaluate the operational efficiency of UAV LiDAR surveying and mapping of rural cadastral maps, using the staff and time invested in this test survey area with the same survey area using the traditional method (GNSS + total station). The workload and efficiency are compared, and the comparison results are shown in table 2 .

\begin{tabular}{|c|c|c|c|}
\hline $\begin{array}{c}\text { Contents of } \\
\text { Work }\end{array}$ & $\begin{array}{c}\text { Traditional } \\
\text { Method } \\
\text { (GNSS + } \\
\text { Total } \\
\text { Station) } \\
\end{array}$ & $\begin{array}{c}\text { LiDAR } \\
\text { Measurement }\end{array}$ & $\begin{array}{c}\text { Efficiency } \\
\text { Ratio of } \\
\text { LiDAR VS } \\
\text { Traditional } \\
\text { Method }\end{array}$ \\
\hline Field survey & $\begin{array}{c}\text { About } 7 \\
\text { days }\end{array}$ & 0.5 days & $\begin{array}{c}\text { About } 14 \\
\text { times }\end{array}$ \\
\hline Housekeeping & $\begin{array}{c}\text { About } 8 \\
\text { days }\end{array}$ & 2 days & $\begin{array}{c}\text { About } 4 \\
\text { times }\end{array}$ \\
\hline Staff input & $\begin{array}{c}\text { About } 20 \\
\text { days }\end{array}$ & 8 people & $\begin{array}{c}\text { About } 2.5 \\
\text { times }\end{array}$ \\
\hline $\begin{array}{l}\text { Comprehensive } \\
\text { per capita } \\
\text { efficiency }\end{array}$ & $\begin{array}{c}13 \text { parcels / } \\
\text { day }\end{array}$ & $\begin{array}{c}101 \text { parcels / } \\
\text { day }\end{array}$ & $\begin{array}{l}\text { About } 7.8 \\
\text { times }\end{array}$ \\
\hline
\end{tabular}

Overall operation efficiency is improved by more than 7 times

Table 2 The comparison of technical efficiency between traditional measurement and UAV LiDAR.

\section{CONCLUSION}

LiDAR measurement technology has the advantages of high efficiency and high accuracy for cadastral survey. This paper focuses on the data acquired by UAV LiDAR, supplemented by the data acquired by vehicle LiDAR, the rural cadastral survey is carried out. This paper analyzes the whole process and accuracy evaluation of cadastral survey with LiDAR survey technology, and verifies the feasibility of technical route and the reliability of product accuracy. In this experiment, the in-plane error of cadastral survey combining UAV LiDAR and vehicleborne LiDAR is about $0.047 \mathrm{~m}$, which is better than $0.050 \mathrm{~m}$ required by the specification, which meets the requirements of the cadastral surveying and mapping. In addition, compared with the traditional cadastral survey method (GNSS + total station), the overall operation efficiency of cadastral survey using LiDAR surveying technology in this experiment is improved by more than 7 times. This experiment can provide theoretical and practical references for the application of LiDAR scanning technology in related fields.

\section{ACKNOWLEDGEMENTS}

The author wishes to thank those who contributed to this experimental project. Thanks to experts and scholars who have provided references for this paper.

\section{REFERENCES}

Bao J., 2019. Research and application of cadastral surveying and mapping based on vehicle LiDAR measurement system. geospatial information.

Cina A , Manzino A M , Manzino G., 2016. Recovery of cadastral boundaries with GNSS equipment. Survey Review, 48(350), 338-346.
Gao Wenxiu, Zhang Yan, et al., 1999. Management of spatiotemporal data of cadastral information system in china. Geo Spatial Information Science.

Abidin H Z , Haroen T S , Adiyanto F H , et al., 2015. On the establishment and implementation of GPS CORS for cadastral surveying and mapping in Indonesia. Survey Review, 47(340), $61-70$.

Independent Multidisciplinary Science Team, 2012. Urban and rural-residential area land uses in Oregon: a synthesis of an IMST technical workshop on watershed functions and salmonid recovery.

In, Su, Lee, et al., 2011. Delineating the natural features of a cadastral shoreline in south korea using airborne laser scanning. Selected Topics in Applied Earth Observations \& Remote Sensing IEEE Journal of 2011.

Laura, Chasmer, Chris, et al., 2014. Investigating laser pulse penetration through a conifer canopy by integrating airborne and terrestrial LiDAR. Canadian Journal of Remote Sensing.

Ussyshkin R V, Theriault L, Sitar M, et al., 2011. Advantages of airborne LiDAR technology in power line asset management. Multi-Platform/Multi-Sensor Remote Sensing and Mapping (M2RSM), 2011 International Workshop on. IEEE.

Thiel C , Baade J, Schmullius C., 2017. Comparison of UAV photograph-based and airborne LiDAR based point clouds over forest from a forestry application perspective. International journal of remote sensing, 38(15-16), 4765-4765.

Zhou Z W , Lv H, 2011. Technology development of UAV recovery system based on laser detection. International Symposium on Photoelectronic Detection and Imaging 2011: Laser Sensing and Imaging; and Biological and Medical Applications of Photonics Sensing and Imaging. International Society for Optics and Photonics.

Zhang J, Li S , Zhang L, et al., 2017. Application and research of uav aerial system in 1:500 rural cadastral mapping. Journal of Geomatics, 42(1), 69-72 and 77. 